Rev. Inst. Flor. v. 29 n. 1 p. $39-55$ jun. 2017

http://dx.doi.org/10.24278/2178-5031.201729103

ISSN impresso 0103-2674/on-line 2178-5031

\title{
ESTRUTURA POPULACIONAL DE 12 ESPÉCIES ARBÓREAS DE DIFERENTES GRUPOS ECOLÓGICOS ${ }^{1}$
}

\author{
POPULATION STRUCTURE OF 12 TREE SPECIES OF \\ DIFFERENT ECOLOGICAL GROUPS
}

\author{
Silvana Cristina Pereira Muniz de SOUZA ${ }^{2,5}$; Flavio Antonio Maës dos SANTOS³; \\ Ricardo Ribeiro RODRIGUES ${ }^{4}$; Carlos Alfredo JOLY3
}

\begin{abstract}
RESUMO - O presente estudo teve como objetivo caracterizar a estrutura populacional de 12 espécies arbóreas de diferentes grupos ecológicos, classificadas de acordo com a tolerância à sombra: i) secundárias iniciais: Alseis floribunda, Bathysa australis, Handroanthus serratifolius, Myrsine hermogenesii; ii) clímax de dossel: Chrysophyllum viride, Quiina glazovii, Tetrastylidium grandifolium, e iii) clímax de subosque: Guapira opposita, Eugenia expansa, Garcinia gardneriana, Inga marginata, Rudgea jasminoides. A área de estudo localiza-se no Parque Estadual de Carlos Botelho, no interior de uma parcela permanente de 10 ha, na qual foram sorteadas 25 subparcelas de $20 \times 20 \mathrm{~m}$. Registramos, das espécies selecionadas, o diâmetro do caule no nível do solo - DAS de todos os indivíduos com altura $>3 \mathrm{~cm}$. As estruturas populacionais foram caracterizadas pela frequência de distribuição de diâmetros, medidas de assimetria das curvas de distribuição, coeficiente de Gini e o ajuste a três modelos: Log-normal, Exponencial Negativa e Weibull. Amostramos 5.410 indivíduos, $90 \%$ apresentaram DAS inferior a 4,8 cm. Todas as espécies apresentaram curvas de distribuições de DAS com assimetria positiva, o modelo Log-normal foi selecionado como o melhor ajuste e todas as espécies apresentaram baixa equabilidade de tamanhos (coeficiente de Gini $>0,5$ ). Somente as espécies clímax de subosque tiveram a distribuição de tamanhos de acordo com o esperado, em formato de "J invertido" e com alta hierarquia de tamanhos. Para as demais espécies, não foi possível estabelecer uma relação direta entre a estrutura de tamanhos e o que é teorizado para o seu grupo ecológico de tolerância à sombra.
\end{abstract}

Palavras-chave: estrutura de tamanho; distribuição diamétrica; classificação sucessional; Mata Atlântica.

\footnotetext{
${ }^{1}$ Parte da tese de doutorado do primeiro autor. Recebido para análise em 26 02.2016. Aceito para publicação em 28.04 .2017$. ${ }^{2}$ Instituto Florestal, Rua do Horto, 931, 02377-000, São Paulo, SP, Brasil.

${ }^{3}$ Universidade Estadual de Campinas, Instituto de Biologia, Departamento de Biologia Vegetal, Caixa Postal 6109, 13081-970, Campinas, SP, Brasil.

${ }^{4}$ Universidade de São Paulo - ESALQ, Departamento de Ciências Biológicas, Laboratório de Ecologia e Restauração Florestal, Avenida Pádua Dias, 11, Caixa

Postal 9,13418-900, Piracicaba, SP, Brasil.

${ }^{5}$ Autor para correspondência: Silvana Cristina Pereira Muniz de Souza - silvana1souza@gmail.com
} 


\begin{abstract}
This study aimed to characterize the population structure of 12 tree species of different ecological groups, classified according shade tolerance: i) initial secondary: Alseis floribunda, Bathysa australis, Handroanthus serratifolius, Myrsine hermogenesii; ii) canopy climax: Chrysophyllum viride, Quiina glazovii, Tetrastylidium grandifolium and iii) understory climax: Eugenia expansa, Garcinia gardneriana, Guapira opposita, Inga marginata, Rudgea jasminoides. The study area is located in the Carlos Botelho State Park, within a 10 ha permanent plot; 25 sub-plots of $20 \times 20 \mathrm{~m}$ were randomly selected. All individuals with height $\geq 3 \mathrm{~cm}$ were tagged and measured for their diameter at ground height. The population structures were characterized by the frequency of distribution of diameters, asymmetry measures of the distribution curves, Gini coefficient and the fit of three models: Log-normal, Exponential Negative and Weibull. We sampled 5,410 individuals, 90\% presented DGB less than $4.8 \mathrm{~cm}$. All species presented DGB distribution curves with positive asymmetry, the Log-normal model was selected with the best fit and all species presented low sizes evenness (Gini coefficient $>0.5$ ). Only the understory climax species had the size distribution according to the expected, in inverted $\mathrm{J}$ curve format and with high hierarchy of sizes. For the other species, we did not found a direct relationship between the size structure and what is theorized for its ecological group of shade tolerance.
\end{abstract}

Keywords: population structure; distribution of diameters; ecological groups; Atlantic Forest.

\section{INTRODUÇÃO}

A estrutura das populações de plantas resulta da ação de fatores bióticos e abióticos sobre as taxas de recrutamento, crescimento e mortalidade de seus membros e de seus antepassados. (Hutchings, 1997). Entre os processos bióticos há os de ordem populacional e os de ordem sinecológica. Do ponto de vista populacional, os fatores genéticos e ecológicos aumentam a diferença de tamanho entre os indivíduos. A herança genética pode manifestar-se por meio de diferenças no tamanho inicial dos indivíduos, nas taxas de crescimento individuais e na habilidade competitiva (Silvertown e Doust, 1993; Hutchings, 1997). Já fatores ecológicos como patógenos, parasitas e herbívoros podem provocar a morte, principalmente de plantas menores (Swaine et al., 1987). Do ponto de vista sinecológico, as clareiras naturais, que se formam pela queda de árvores liberam recursos antes indisponíveis, promovendo a germinação de muitas sementes e o crescimento de plântulas e jovens árvores estabelecidas (Swaine et al., 1987; Zavala et al., 2007).

Entre os fatores abióticos podemos destacar as características do solo como textura, fertilidade e disponibilidade hídrica, além da temperatura e luminosidade (Barbour et al., 1987). Nas florestas tropicais, a radiação que atinge o solo da floresta em áreas sob dossel contínuo corresponde a somente $1-3 \%$ da radiação total incidente sobre o dossel (Chazdon, 1988), consistindo na condição abiótica com maior influência sobre o crescimento das plântulas (Baskin e Baskin, 1998). Considerando o fator luminosidade, as espécies podem ser separadas em tolerantes à sombra e intolerantes à sombra. Espécies tolerantes à sombra podem germinar, crescer e sobreviver em condições de pouca luminosidade, enquanto espécies intolerantes à sombra necessitam de ambiente iluminado para germinação e/ou estabelecimento das plântulas (Lee, 1996; Whitmore, 1996; Baskin e Baskin, 1998). Em florestas tropicais, a abertura de clareiras é a oportunidade para o estabelecimento das espécies intolerantes à sombra, sendo a dinâmica de clareiras considerada peça-chave para o entendimento da estrutura e dinâmica destas florestas (Hubbell e Foster, 1986; Lima, 2005). Segundo muitos autores, a regeneração natural que ocorre em seu interior influencia importantes parâmetros das comunidades florestais, como composição (Pearson et al., 2003), distribuição e riqueza de espécies (Denslow, 1995), além dos processos de sucessão secundária (Whitmore, 1990). 
Uma ferramenta frequentemente utilizada para representar a estrutura de uma floresta é a distribuição dos indivíduos de espécies arbóreas em classes de tamanho (Whitmore, 1984; Harper, 1977). O formato da curva de distribuição de populações de espécies arbóreas em classes de tamanho também tem sido largamente utilizado para interpretar as características das populações e classificar as espécies em categorias ou grupos ecológicos de tolerância à sombra (Panetta, 1979; Hartshorn, 1980; Zavala et al., 2007). Na ausência de grandes perturbações, no interior de florestas maduras, espera-se, na estrutura de espécies tolerantes à sombra, maior número de jovens em relação aos adultos (Hubbell e Foster, 1987), em decorrência da capacidade de os indivíduos jovens sobreviverem por longos períodos no subosque da floresta e beneficiarem-se de eventuais aumentos na intensidade luminosa. Sendo assim, as espécies clímax de subosque apresentam distribuição com predominância de indivíduos nas classes menores e poucos nas maiores (Solbrig, 1981; Rubin et al., 2006). Essa forma de distribuição poderia ser representada por uma curva do tipo "J invertido" e estaria associada a tolerância à sombra (Knight, 1975; Wright et al., 2003). Já as espécies clímax de dossel e emergentes geralmente apresentam distribuição dos indivíduos em classes de tamanho variável e flutuante, do tipo bimodal ou multimodal, com algumas classes pouco frequentes em relação às demais (Gentry e Terborgh, 1990; Poorter et al., 1996; Zavala et al., 2007; Nunes e Petrere Júnior, 2012).

Para as espécies que necessitam de luz nos estádios iniciais, secundárias iniciais e pioneiras, há a expectativa de que a regeneração seja infrequente, devido à imprevisibilidade de locais adequados para que esta ocorra, dependendo da abertura de clareiras, e, portanto, o número de indivíduos pequenos pode ser muito baixo (Whitmore, 1984). Desse modo, as espécies intolerantes à sombra podem apresentar curvas de distribuição dos indivíduos em classes de tamanho do tipo sigmoidal ou normal (Panetta, 1979; Bongers et al., 1988; Poorter et al., 1996), já que seu recrutamento estaria associado a grandes perturbações (Hubbell, 1979; Hubbell e Foster, 1987).
Essas diferentes formas de distribuição dos indivíduos em classes de tamanho representariam estratégias ecológicas contrastantes, ou extremos de um gradiente de resposta à luz. O estudo de populações de espécies arbóreas, a partir da distribuição dos indivíduos em classes de tamanho, pode servir de base para a construção inicial de hipóteses, modelos e predições. Pode, também, oferecer uma contribuição importante para planos de manejo e recuperação.

Para avaliar a distribuição de frequências de diâmetros utilizamos distribuições de probabilidade e o coeficiente de Gini. As distribuições de probabilidade ou modelos que apresentam bons ajustes aos dados de diâmetro são, frequentemente, os modelos Exponencial Negativo, Weibull e Log-normal (Bliss e Reinker, 1964; Hafley e Schreuder, 1977). O melhor ajuste ao modelo Exponencial Negativo é esperado quando a mortalidade e crescimento são independentes do tamanho de cada indivíduo (Muller-Landau et al., 2006), o que significa que o crescimento e mortalidade são eventos aleatórios independentes das características de cada indivíduo, assim como os distúrbios (Cox e Oakes, 1984; Lima et al., 2015). Já o modelo Weibull é esperado quando a mortalidade é constante, mas o crescimento se dá em função do tamanho da planta (Muller-Landau et al., 2006). No entanto, se considerarmos que as taxas de crescimento variam no tempo de forma aditiva, o melhor ajuste à distribuição diamétrica será o modelo Log-normal (Otto e Day, 2007). Com o cálculo do coeficiente de Gini obtém-se o grau de hierarquia de tamanho de cada população, independente da utilização de classes de tamanho, ou seja, não se depende da escolha do intervalo de classes e aí reside a grande vantagem desta avaliação (Feeley et al., 2007).

Nesse contexto, este estudo teve como objetivo caracterizar a estrutura populacional de 12 espécies arbóreas, com diferentes graus de tolerância à sombra (secundárias iniciais, clímax de dossel e clímax de subosque) a partir das distribuições de frequência de diâmetros e o coeficiente de Gini, tendo como expectativa que as diferenças no grau de tolerância reflitam no tipo de distribuição probabilística de tamanhos, ou seja, à medida que se aumenta a tolerância à sombra, as curvas de distribuição de diâmetros apresentarão maior desigualdade e maior hierarquia. 


\section{MATERIAL E MÉTODOS}

\section{1 Área de Estudo}

A área escolhida para o estudo localiza-se no município de Sete Barras, região sul do estado de São Paulo, sobre a vertente atlântica da Serra de Paranapiacaba, no interior do Parque Estadual de Carlos Botelho - PECB (240ㅇ' a $24^{\circ} 15^{\prime}$ S, $47^{\circ} 45^{\prime}$ a $\left.48^{\circ} 10^{\prime} \mathrm{W}\right)$. Nessa área, a vegetação é classificada como Floresta Ombrófila Densa Submontana (cota de $300 \mathrm{~m}$ ) (Instituto Brasileiro de Geografia e Estatatística - IBGE, 2012).

$\mathrm{O}$ estudo foi conduzido no interior de uma parcela permanente de 10 ha localizada na unidade geomorfológica conhecida como Planalto de Guapiara, que apresenta clima quente úmido sem estiagem, classificada como Cfa segundo o sistema de Köppen (Köppen, 1948), com temperaturas inferiores a $18{ }^{\circ} \mathrm{C}$ no mês mais frio e superiores a $22{ }^{\circ} \mathrm{C}$ no mês mais quente. A área é caracterizada, predominantemente, por períodos de excedente hídrico, com precipitação média anual de $1.582 \mathrm{~mm}$ (Departamento de Águas e Energia Elétrica - DAEE, 2016), concentrando-se nos meses de outubro a março (Rodrigues, 2006). No interior da parcela permanente do PECB, foram encontrados os seguintes tipos de solo: Cambissolos Háplicos Tb distróficos latossólicos, Cambissolos Háplicos Tb distróficos rasos, Gleissolos Háplicos Tb distróficos típicos e Neossolos Flúvicos. O solo Cambissolo é predominante na parcela, os demais tipos restringem-se a pequenas manchas (Soares Junior et al., 2004).

No interior da parcela de 10 ha, foram sorteadas 25 subparcelas, dentre as 256 subparcelas dos 10 ha da Parcela Permanente, totalizando 1 ha de área amostral. Nas subparcelas sorteadas, prevaleceram as situações de subosque e pequenas clareiras, e entre estas situações não foram encontradas diferenças significativas de luminosidade (Muniz, 2004) . Segundo Lima (2005), clareiras pequenas se formam em consequência da queda de emaranhados de lianas e/ou cipós, da queda de galhos ou da desintegração gradual da copa de uma árvore morta em pé. Geralmente, a queda isolada de árvores não gera grandes clareiras, que comumente são formadas a partir de deslizamentos em encostas, furacões, ou mesmo a queda associada de várias árvores. No período anterior e durante o estudo, não foram observadas clareiras grandes nas subparcelas sorteadas e proximidades.
Para o sorteio das subparcelas foram consideradas apenas as que apresentavam o mesmo tipo de solo, Cambissolo Háplico $\mathrm{Tb}$ distrófico latossólico, para evitar variabilidade entre subparcelas. Nestas unidades amostrais, todos os indivíduos das espécies selecionadas, com altura $>3 \mathrm{~cm}$, foram marcados com fita plástica numerada, tiveram registrado o DAS (diâmetro do caule no nível do solo) utilizando-se um paquímetro (precisão de $0,1 \mathrm{~mm}$ ) ou uma fita métrica para medir o perímetro, no caso de indivíduos de maior diâmetro.

\subsection{Espécies Estudadas}

Selecionamos 12 espécies classificadas em três grupos ecológicos (Tabela 1). A escolha das espécies baseou-se no levantamento fitossociológico realizado na parcela permanente do PECB (Rodrigues, 2006). Os critérios utilizados para a escolha das espécies foram abundância, facilidade de identificação em todos os estádios de desenvolvimento e por serem representantes dos seguintes grupos ecológicos, com base na tolerância à sombra: i) secundárias iniciais, ii) clímax de dossel e iii) clímax de subosque.

As características ecológicas e biológicas de cada espécie foram consultadas em diferentes fontes bibliográficas e observações de campo (Tabela 1). Nos casos em que ocorreu discordância entre os diversos autores ou ausência de informações sobre a classificação sucessional, prevaleceram as observações de campo.

\subsection{Análise dos Dados}

Realizamos a análise estatística descritiva da distribuição dos diâmetros de cada espécie para verificar as medidas de assimetria das curvas de distribuição. Segundo Spiegel e Stephens (2009), a maneira mais simples de verificar a grandeza e o sinal de assimetria de uma curva é por meio da comparação das medidas de tendências central: média aritmética $(\chi)$ e moda $\left(\mathrm{M}_{\mathrm{o}}\right)$, da seguinte maneira: $\mu-M_{o}=0$ - assimetria nula ou distribuição simétrica; $\mu-M_{0}<0$ - assimetria negativa ou à esquerda; $\mu-M_{o}>0-$ assimetria positiva ou à direita. 
SOUZA, S.C.P.M. et al. Estrutura populacional de 12 espécies arbóreas.

Tabela 1. Espécies selecionadas para o estudo da estrutura populacional no Parque Estadual de Carlos Botelho, Sete Barras, SP, com a classificação sucessional e as referências de cada classificação.

Table 1. Species selected for the study of the population structure in the Carlos Botelho State Park, Sete Barras, SP, with the successional classification and the references of each classification.

\begin{tabular}{|c|c|c|}
\hline Espécie & Classificação sucessional & Referência \\
\hline Alseis floribunda Schott & Secundária & Bernacci et al. (2006) \\
\hline Bathysa australis (A.St.-Hill.) K.Schum. & Secundária inicial & Leite e Rodrigues (2008) \\
\hline Handroanthus serratifolius (Vah) S.Grose & Secundária inicial & Gandolfi (1991) \\
\hline $\begin{array}{l}\text { Myrsine hermogenesii (Jung-Mend. \& Bernacci) M.F. } \\
\text { Freitas \& Kin.-Gouv.) }\end{array}$ & Secundária inicial & Bernacci et al. (2006) \\
\hline Chrysophyllum viride Mart. \& Eichler & Típica de dossel & Souza et al. (2014) \\
\hline Quiina glazovii Engl. & Secundária tardia & Catarino et al. (2006) \\
\hline Tetrastylidium grandifolium (Baill.) Sleumer & Típica de dossel & $\begin{array}{l}\text { Sztutman e Rodrigues } \\
\text { (2002) }\end{array}$ \\
\hline Eugenia expansa Spring ex Mart. & Clímax & Oliveira-Filho et al. (2004) \\
\hline Garcinia gardneriana (Planch. \& Triana) Zappi & $\begin{array}{l}\text { Tolerante à sombra de } \\
\text { subosque }\end{array}$ & $\begin{array}{l}\text { Tabarelli e Mantovani } \\
\text { (1997) }\end{array}$ \\
\hline Guapira opposita (Vell.) Reitz & Tolerante à sombra & Oliveira-Filho et al. (2004) \\
\hline Inga marginata Willd. & Tolerante à sombra & Oliveira-Filho et al. (2004) \\
\hline Rudgea jasminoides (Cham.) Müll.Arg. & Tolerante à sombra & Oliveira-Filho et al. (2004) \\
\hline
\end{tabular}

Testamos o ajuste de três distribuições de densidade probabilística selecionadas a partir dos pressupostos teóricos de cada uma e por serem empregadas para descrever a variação de dados de diâmetro de populações arbóreas: distribuição Exponencial Negativa (Muller-Landau et al., 2006), Log-normal (Chen, 2004) e Weibull (Bailey e Dell, 1973). A distribuição Exponencial Negativa é frequentemente usada nas distribuições diamétricas de árvores (Lima, 2013), possui apenas um parâmetro que controla a inclinação da distribuição que é decrescente. A distribuição Log-normal é bastante comum na natureza e frequentemente usada para descrever valores contínuos de tamanho (Bolker, 2008). Na distribuição Log-normal, é possível estabelecer uma relação entre o grau de desigualdade da distribuição e os parâmetros da curva, esta distribuição se torna mais semelhante à curva normal à medida que o desvio-padrão diminui e mais próxima do formato de "J invertido" com o aumento do desvio-padrão (Otto e Day, 2007). A distribuição Weibull também é uma distribuição flexível, pois pode tanto se aproximar do formato de uma curva normal, quanto de uma distribuição Exponencial Negativa (Crawley, 2007; Otto e Day, 2007).

Utilizamos um procedimento de seleção de modelos para verificar qual tipo de distribuição de densidade probabilística melhor se ajustava aos dados de distribuição de diâmetros de cada espécie. Selecionamos os modelos a partir do Critério de Informação de Akaike - AIC, que representa uma ponderação entre o número de parâmetros e o ganho em explicabilidade do modelo, com base no cálculo da verossimilhança (Crawley, 2007). O valor estimado pelo AIC representa a distância do modelo selecionado a um "modelo verdadeiro" (Hobbs e Hilborn, 2006). Desse modo, quanto menor o AIC, melhor é considerado o modelo, destacando-se que para dois modelos serem considerados diferentes é necessário que o $\Delta \mathrm{AIC} \geq 2$ (Bolker, 2008). Para a seleção de modelos utilizamos o software R versão 3.3.1 (R Development Core Team, 2016), empregando o pacote bbmle - "Tools for general maximum likelihood estimation" (Bolker, 2016). 
Calculamos o coeficiente de Gini para os dados de diâmetro dos indivíduos. O coeficiente de Gini é uma medida de concentração de tamanho ou de biomassa numa dada população, variando de 0 a 1 , sendo que, quanto mais próximo de 1, maior é a desigualdade de tamanhos (Weiner e Solbrig, 1984). Para testar a igualdade entre as hierarquias das espécies e dos grupos ecológicos realizamos reamostragens de bootstrap com 1.000 repetições. Para os referidos cálculos $\begin{array}{lllll}\text { utilizamos } & \mathrm{o} & \text { software } \mathrm{R} & \text { versão } & 3.3 .1\end{array}$ (R Development Core Team, 2016).

\section{RESULTADOS}

O levantamento das 12 espécies resultou em 5.410 indivíduos; deste total, 778 indivíduos foram agrupados como secundárias iniciais,
1.769 indivíduos como clímax de dossel e 2.863 como indivíduos clímax de sobosque. A distribuição das espécies em classes de tamanho resultou em 90\% dos indivíduos com DAS inferior a $4,8 \mathrm{~cm}$.

A comparação dos valores obtidos a partir da estatística descritiva indicou que as distribuições diamétricas de todas as espécies são assimétricas positivas, já que os valores da média foram maiores do que a moda (Tabela 2), indicando alta concentração no lado esquerdo das distribuições diamétricas com a cauda da curva, com poucas observações proporcionalmente ao tamanho da amostra, se estendendo ao lado direito. A assimetria positiva das distribuições de frequência de diâmetros é uma característica das curvas com o formato de "J invertido". Não foram observadas distribuições com curvas no formato normal ou multimodal.

Tabela 2. Descritores estatísticos da distribuição da variável diâmetro à altura do solo - DAS para as 12 espécies estudadas no Parque Estadual de Carlos Botelho, Sete Barras, SP. Número de indivíduos amostrados (Ni), desvio-padrão $(\sigma)$, média $(\mu)$, moda $\left(M_{0}\right)$, comparação da medida de tendência central $\left(\mu-M_{0}\right)$ e sinal da assimetria da curva ( + assimetria positiva).

Table 2. Statistical descriptors of the distribution of the variable diameter at ground height - DGH for the 12 species studied in the Carlos Botelho State Park, Sete Barras, SP. Number of individuals sampled (Ni), standard deviation $(\sigma)$, mean $(\mu)$, mode $\left(M_{o}\right)$, comparison of the central tendency measure $\left(\mu-M_{o}\right)$ and curve asymmetry signal (+ positive asymmetry).

\begin{tabular}{|c|c|c|c|c|c|c|}
\hline \multirow{2}{*}{ Espécies } & \multirow{2}{*}{$\mathrm{Ni}$} & \multicolumn{3}{|c|}{ Descritores } & \multirow{2}{*}{$\mu-$ Mo } & \multirow{2}{*}{ Assimetria da curva } \\
\hline & & $\sigma$ & $\mu$ & Mo & & \\
\hline Alseis floribunda & 91 & 5,2 & 2,1 & 0,3 & 1,8 & + \\
\hline Bathysa australis & 124 & 6,6 & 3,6 & 0,4 & 3,2 & + \\
\hline Handroanthus serratifolius & 96 & 9,5 & 6,0 & 1,2 & 4,8 & + \\
\hline Myrsine hermogenesii & 467 & 3,0 & 1,0 & 0,2 & 0,8 & + \\
\hline Chrysophyllum viride & 113 & 16,5 & 6,1 & 0,7 & 5,4 & + \\
\hline Quiina glaziovii & 127 & 6,5 & 3,2 & 0,5 & 2,7 & + \\
\hline Tetrastylidium grandifolium & 1529 & 4,5 & 1,3 & 0,4 & 0,9 & + \\
\hline Eugenia expansa & 324 & 2,3 & 2,0 & 0,9 & 1,1 & + \\
\hline Garcinia gardneriana & 514 & 4,5 & 3,3 & 0,3 & 3,0 & + \\
\hline Guapira opposita & 957 & 4,7 & 3,1 & 0,6 & 2,5 & + \\
\hline Inga marginata & 393 & 1,5 & 0,7 & 0,2 & 0,5 & + \\
\hline Rudgea jasminoides & 675 & 2,8 & 1,2 & 0,3 & 0,9 & + \\
\hline
\end{tabular}


As distribuições diamétricas das espécies secundárias iniciais e clímax de dossel não se assemelharam aos modelos esperados para os grupos ecológicos destas espécies (Figura 1). Somente o grupo das espécies clímax de subosque tiveram a distribuição de tamanhos de acordo com o esperado, em formato de "J invertido" (Figura 1).

As distribuições diamétricas de todas as espécies, independentemente de seu grupo ecológico, apresentaram melhor ajuste aos dados quando empregamos a distribuição Log-normal, sendo a distribuição de densidade probabilística selecionada (Tabela 3). A distribuição de Weibull foi o segundo modelo com melhor ajuste e a distribuição Exponencial Negativa, em terceiro lugar para quase todas as espécies, a única exceção foi a espécie Eugenia expansa em que a distribuição Exponencial Negativa foi o segundo modelo com melhor ajuste e a distribuição de Weibull, o terceiro (Tabela 3).
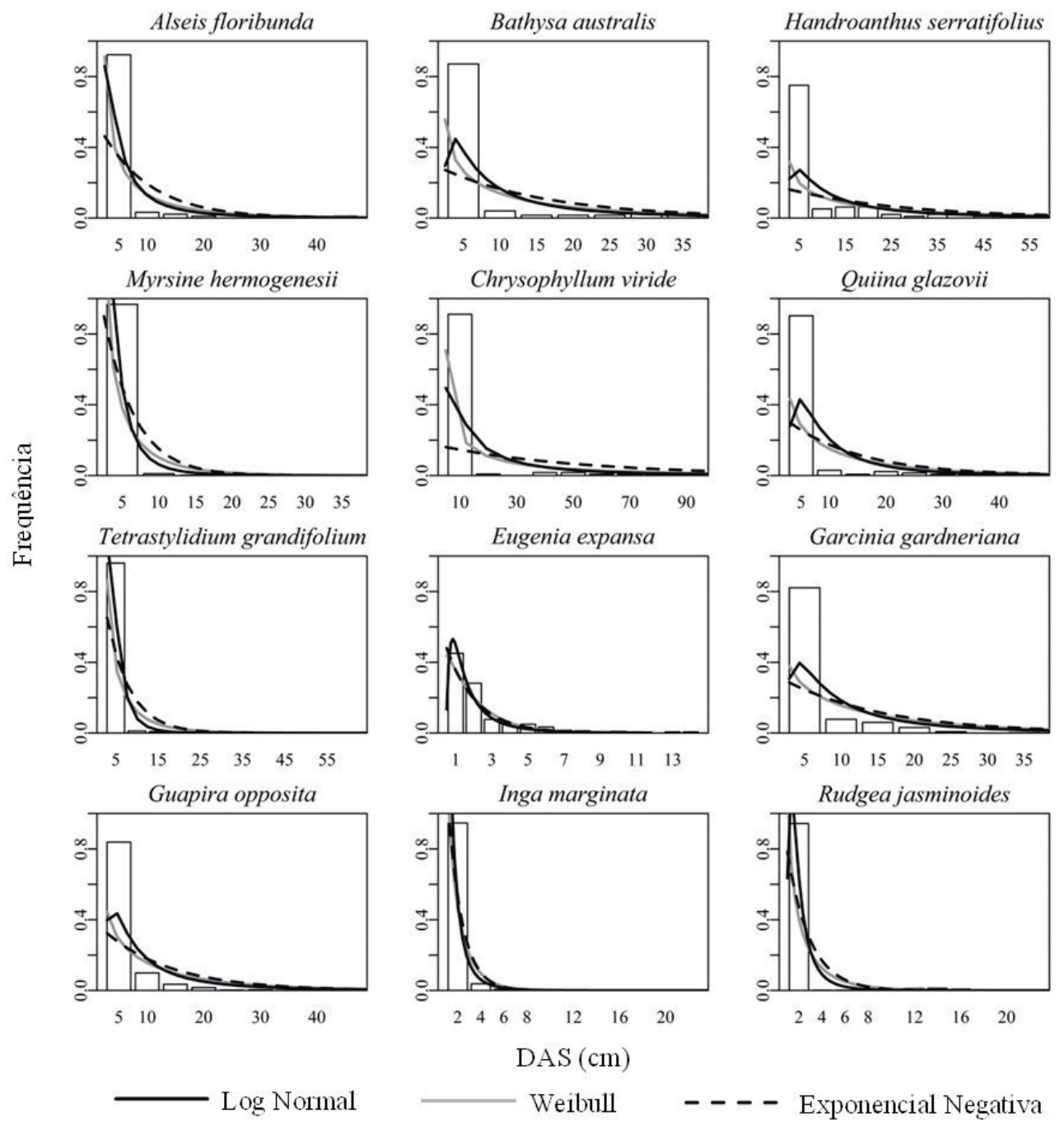

Figura 1. Distribuição de frequência de diâmetros e o seus respectivos ajustes aos modelos Log-normal, Weibull e Exponencial Negativa das espécies estudadas no Parque Estadual de Carlos Botelho, Sete Barras, SP. Note que a distribuição Log-normal apresentou melhor ajuste.

Figure 1. Frequency distribution of diameters and their respective fits to the Log-normal, Weibull and Exponential Negative models of the studied species in the Carlos Botelho State Park, Sete Barras, SP. Notice that the Log-normal distribution presented the best fit. 
Tabela 3. Resultado da seleção dos modelos de distribuição probabilística ajustados às distribuições da variável diâmetro à altura do solo - DAS das 12 espécies estudadas no Parque Estadual de Carlos Botelho, Sete Barras, SP. $\mathrm{O}$ modelo com o $\triangle \mathrm{AIC}$ igual a zero é aquele com o melhor ajuste. AIC = Critério de Informação de Akaike; $\mathrm{gl}=$ graus de liberdade; $\triangle \mathrm{AIC}=$ diferença entre os valores de $\mathrm{AIC}$ dentre os modelos ajustados.

Table 3. Results of the selection of probabilistic distribution models adjusted to the distributions of the variable diameter at ground height - DGH of the 12 species studied in the Carlos Botelho State Park, Sete Barras, SP. The model with $\triangle \mathrm{AIC}$ equal to zero is the one with the best fit. $\mathrm{AIC}=$ Akaike Information Criterion; $\mathrm{gl}=$ degrees of freedom; $\triangle \mathrm{AIC}=$ difference among AIC values among adjusted models.

\begin{tabular}{l|ccc|ccc|ccc}
\hline \multirow{2}{*}{ Espécies } & \multicolumn{7}{c}{ Modelos } \\
\cline { 2 - 10 } & \multicolumn{3}{|c|}{ Log-normal } & \multicolumn{3}{c}{ Weibull } & \multicolumn{3}{c}{ Exponencial negativa } \\
\hline \multirow{3}{*}{ Alseis floribunda } & AIC & gl & $\Delta$ AIC & AIC & gl & AIC & AIC & gl & $\Delta$ AIC \\
\cline { 2 - 9 } Bathysa australis & 253,3 & 2 & 0,0 & 285,1 & 2 & 31,8 & 315,5 & 1 & 62,2 \\
Handroanthus serratifolius & 511,2 & 2 & 0,0 & 543,3 & 2 & 32,1 & 565,8 & 1 & 54,6 \\
Myrsine hermogenesii & 494,2 & 2 & 0,0 & 520,2 & 2 & 26,1 & 536,8 & 1 & 42,6 \\
Chrysophyllum viride & 473,5 & 2 & 0,0 & 780,5 & 2 & 306,9 & 941,4 & 1 & 467,8 \\
Quiina glaziovii & 472,8 & 2 & 0,0 & 528,1 & 2 & 55,3 & 637,3 & 1 & 164,4 \\
Tetrastylidium grandifolium & 494,5 & 2 & 0,0 & 559,6 & 2 & 65,1 & 574,2 & 1 & 79,7 \\
Eugenia expansa & 1903,6 & 2 & 0,0 & 3359,1 & 2 & 1445,6 & 3900,7 & 1 & 1997,1 \\
Garcinia gardneriana & 1036,8 & 2 & 0,0 & 1094 & 2 & 57,2 & 1092,9 & 1 & 56,1 \\
Guapira opposita & 2126,1 & 2 & 0,0 & 2233,8 & 2 & 107,7 & 2255,2 & 1 & 129,1 \\
Inga marginata & 3817,0 & 2 & 0,0 & 4086,2 & 2 & 269,2 & 4159,5 & 1 & 342,4 \\
Rudgea jasminoides & 388,0 & 2 & 0,0 & 526,9 & 2 & 138,8 & 540,6 & 1 & 152,5 \\
& 959,6 & 2 & 0,0 & 1449,2 & 2 & 489,6 & 1565,8 & 1 & 606,2 \\
\hline
\end{tabular}

Os valores de coeficiente de Gini para os diâmetros foram superiores a 0,5 , indicando alta desigualdade entre nos diâmetros (alta hierarquia) para todas as espécies (Tabela 4). As espécies com maior desigualdade nos diâmetros foram Chrysophyllum viride, Myrsine hermogenesii e Alseis floribunda. Por outro lado, a espécie Eugenia expansa, apresentou a menor desigualdade nos tamanhos (Tabela 4). A reamostragem bootstrap do coeficiente de Gini das espécies evidenciou $C$. viride como diferente das demais espécies, exceto com $M$. hermogenesii e A. floribunda; e a espécie $E$. expansa diferente de todas espécies menos Quina glaziovii e Inga marginata (Figura 2). Ao considerar os grupos ecológicos, os maiores valores do coeficiente de Gini foram os das espécies secundárias iniciais, seguidos das espécies de dossel e por último as espécies de sobosque (Tabela 5). A partir da reamostragem bootstrap encontramos diferenças significativas entre o grupo das espécies de subosque e os demais grupos; não observamos diferenças significativas entre o grupo das clímax de dossel e as secundárias iniciais (Figura 3).

\section{DISCUSSÃO}

Todas as espécies estudadas apresentaram em comum elevada concentração de indivíduos na primeira classe de tamanho com diminuição drástica do número de indivíduos na passagem da primeira para as demais classes, constatado a partir da assimetria positiva na distribuição diamétrica de todas as espécies. Harper (1977) e Silvertown e Doust (1993) denominaram essa severa redução da primeira para as demais classes de "efeito gargalo". Se a primeira classe de tamanho é realmente uma classe na qual se acumulam indivíduos, a passagem para a próxima classe estaria condicionada a alguma alteração nas condições do microssítio, especialmente a luminosidade (Lima, 2005). A distribuição dos indivíduos em estruturas do tipo "J-invertido", indica um balanço positivo entre recrutamento e mortalidade, que só ocorre quando os indivíduos menores substituem, sucessivamente, os indivíduos adultos na população (Meira-Neto e Martins, 2003; Bernasol e Lima-Ribeiro, 2010; Souza et al., 2012). 
SOUZA, S.C.P.M. et al. Estrutura populacional de 12 espécies arbóreas.

Tabela 4. Comparação entre as distribuições de coeficiente de Gini gerados por bootstrap calculados a partir da distribuição dos diâmetros à altura do solo - DAS das 12 espécies estudadas no Parque Estadual de Carlos Botelho, Sete Barras, SP. As letras iguais indicam que não há diferença estatística entre as distribuições de coeficiente de Gini. $\mathrm{G}=$ coeficiente de Gini calculado a partir dos dados de distribuição dos DAS; Gm = mediana; Intervalo de confiança $=$ quantis $2,5 \%$ e 97,5\% da distribuição de coeficientes de Gini gerados por bootstrap.

Table 4. Comparison among the distributions of Gini coefficient generated by bootstrap calculated from the distribution of soil diameters - DAS of the 12 species studied in the Carlos Botelho State Park, Sete Barras, SP. Equal letters indicate that there is no statistical difference between the distributions of Gini coefficient. $\mathrm{G}=\mathrm{Gini}$ coefficient calculated from DAS distribution data; $\mathrm{Gm}=$ median; Confidence interval $=2.5 \%$ and $97.5 \%$ of the distribution of Gini coefficients generated by bootstrap.

\begin{tabular}{lcccc}
\hline \multirow{2}{*}{ Espécies } & \multirow{2}{*}{$\mathrm{G}$} & $\mathrm{Gm}$ & \multicolumn{2}{c}{ Intervalo de confiança } \\
\cline { 4 - 5 } & & $0,6927 \mathrm{a}, \mathrm{b}$ & 0,5594 & $0,5 \%$ \\
\hline Alseis floribunda & 0,7072 & $0,6533 \mathrm{a}$ & 0,5726 & 0,6955 \\
Bathysa australis & 0,6569 & $0,6542 \mathrm{a}$ & 0,5984 & 0,7021 \\
Handroanthus serratifolius & 0,6616 & $0,6972 \mathrm{a}, \mathrm{b}$ & 0,6107 & 0,7491 \\
Myrsine hermogenesii & 0,6991 & $0,7990 \mathrm{~b}$ & 0,7232 & 0,8225 \\
Chrysophyllum viride & 0,8044 & $0,6255 \mathrm{a}, \mathrm{c}$ & 0,5303 & 0,6853 \\
Quiina glaziovii & 0,6322 & $0,6577 \mathrm{a}$ & 0,6025 & 0,7017 \\
Tetrastylidium grandifolium & 0,6611 & $0,5088 \mathrm{c}$ & 0,4756 & 0,5405 \\
Eugenia expansa & 0,5106 & $0,5955 \mathrm{a}$ & 0,5729 & 0,6153 \\
Garcinia gardneriana & 0,5960 & $0,6153 \mathrm{a}$ & 0,5933 & 0,6361 \\
Guapira opposita & 0,6166 & $0,5733 \mathrm{a}, \mathrm{c}$ & 0,5036 & 0,6370 \\
Inga marginata & 0,5768 & $0,6273 \mathrm{a}$ & 0,5692 & 0,6680 \\
Rudgea jasminoides & 0,6298 & & & \\
\hline
\end{tabular}

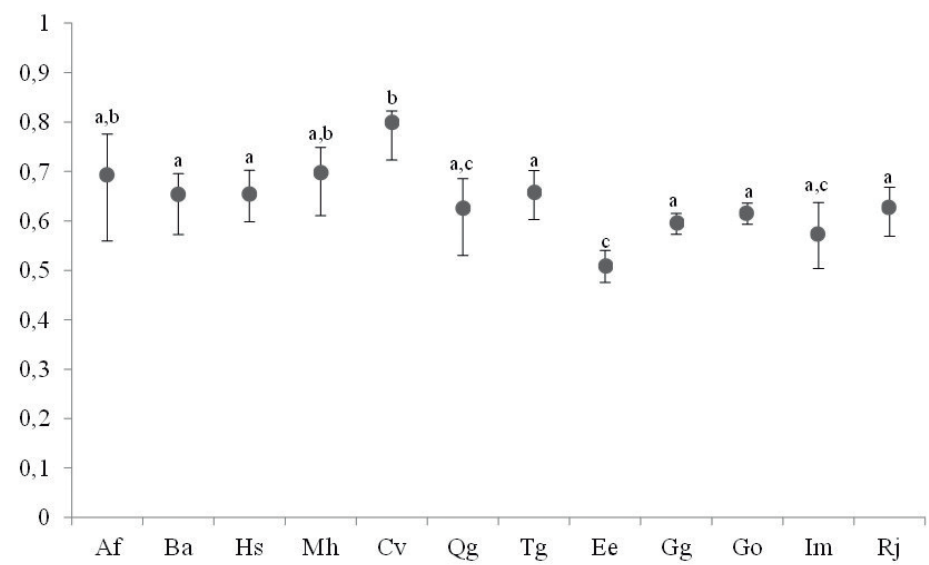

Figura 2. Distribuição das medianas dos coeficientes de Gini e respectivos intervalos de confiança das 12 espécies estudadas no Parque Estadual de Carlos Botelho, Sete Barras, SP. Letras iguais indicam que não há diferença estatística entre os valores. Af = Alseis floribunda, Ba = Bathysa australis, Hs = Handroanthus serratifolius, $\mathrm{Mh}=$ Myrcine hermogenesii, $\mathrm{Cv}=$ Chrysophyllum viride, $\mathrm{Qg}=$ Quiina glazovii, $\mathrm{Tg}=$ Tetrastylidium grandifolium, $\mathrm{Ee}=$ Eugenia expansa, $\mathrm{Gg}=$ Garcinia gardneriana, Go = Guapira opposita, Im = Inga marginata e $\mathrm{Rj}=$ Rudgea jasminoides .

Figure 2. Distribution of the medians of Gini coefficients and respective confidence intervals of the 12 species studied in the Carlos Botelho State Park, Sete Barras, SP. Equal letters indicate that there is no statistical difference between values. 
SOUZA, S.C.P.M. et al. Estrutura populacional de 12 espécies arbóreas.

Tabela 5. Comparação entre as distribuições de coeficiente de Gini gerados por bootstrap calculados a partir da distribuição dos diâmetros à altura do solo - DAS das 12 espécies estudadas por grupos ecológicos no Parque Estadual de Carlos Botelho, Sete Barras, SP. As letras iguais indicam que não há diferença estatística entre as distribuições de coeficiente de Gini. $\mathrm{G}=$ coeficiente de Gini calculado a partir dos dados de distribuição dos DAS; Gm = mediana; Intervalo de confiança = quantis 2,5\% e 97,5\% da distribuição de coeficientes de Gini gerados por bootstrap.

Table 5. Comparison among the distributions of Gini coefficient generated by bootstrap calculated from the diameter at ground height - DGH of the 12 species studied by ecological groups in the Carlos Botelho State Park, Sete Barras, SP. Equal letters indicate that there is no statistical difference among the distributions of Gini coefficient. $\mathrm{G}=$ Gini coefficient calculated from DGH distribution data; Gm = median; Confidence interval $=2.5 \%$ and $97.5 \%$ of the distribution of Gini coefficients generated by bootstrap.

\begin{tabular}{lcccc}
\hline \multirow{2}{*}{ Grupos ecológicos } & \multirow{2}{*}{$\mathrm{G}$} & $\mathrm{Gm}$ & \multicolumn{2}{c}{ Intervalo de confiança } \\
\cline { 4 - 5 } & & $0,7323 \mathrm{a}$ & 0,69218 & $2,5 \%$ \\
\hline Secundárias iniciais & 0,7515 & $0,7220 \mathrm{a}$ & 0,682345 & 0,7640 \\
Clímax de dossel & 0,7222 & $0,6557 \mathrm{~b}$ & 0,643759 & 0,7557 \\
Clímax de subosque & 0,6482 & 0,6680 \\
\hline
\end{tabular}

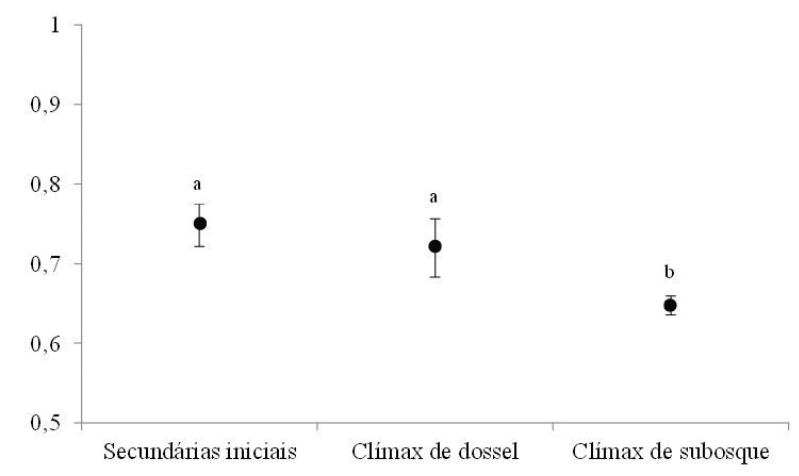

Figura 3. Distribuição das medianas dos coeficientes de Gini e respectivos intervalos de confiança dos grupos ecológicos selecionados no Parque Estadual de Carlos Botelho, Sete Barras, SP. Letras iguais indicam que não há diferença estatística entre os valores.

Figure 3. Distribution of the medians of Gini coefficients and respective confidence intervals of the selected ecological groups in the Carlos Botelho State Park, Sete Barras, SP. Equal letters indicate that there is no statistical difference between values.

As espécies do grupo das secundárias iniciais (Alseis floribunda, Bathysa australis, Handroanthus serratifolia e Myrsine hermogenesii) não apresentaram os modelos sigmoidal ou normal esperado para a distribuição dos indivíduos em classes de tamanho (Bongers et al., 1988; Oliveira-Filho et al., 1996; Poorter et al., 1996; Lobo-Faria, 1998), os quais exibem estruturas de tamanho semelhantes e predominância de indivíduos nas classes intermediárias. Esta estrutura, segundo Knight (1975) e Hubbell (1979), seria resultante da reduzida capacidade de sobrevivência de indivíduos jovens sob sombreamento, não formando banco de plântulas ou de jovens. Os efeitos do sombreamento para as espécies estudadas provavelmente refletiram na baixa densidade relativa de $A$. floribunda, $B$. australis e H. serratifolius. Um evento reprodutivo em data próxima à coleta de dados (observação pessoal) da espécie $M$. hermogenesii determinou a alta densidade de indivíduos recrutados recentemente, na qual, do total de indivíduos, 70\% apresentaram DAS menor que $0,5 \mathrm{~cm}$, o que justifica a alta densidade dessa espécie em relação às demais do grupo das secundárias iniciais. Desse modo, a época de realização do censo pode influenciar na determinação da estrutura de tamanhos, assim como, fatores de periodicidade da reprodução (anual ou supra-anual). 
Quanto às espécies do grupo clímax de dossel, a estrutura populacional esperada também não foi observada. Geralmente, nas espécies de dossel e emergentes, a distribuição dos indivíduos em classes de tamanho é do tipo bimodal ou multimodal (Zavala et al., 2007), caracterizada por uma estrutura de tamanhos variável e flutuante, com algumas classes pouco frequentes em relação às demais. Essas classes estariam associadas a uma variação temporal nas taxas de sobrevivência e recrutamento, devido a grandes perturbações e/ou diferentes demandas de luz durante a ontogenia dos indivíduos (Clark e Clark, 1987). Algumas espécies de dossel demandam repetidos eventos de abertura de clareira para o recrutamento progressivo de indivíduos nas maiores classes de tamanho, até atingirem o dossel da floresta.

A rejeição da hipótese inicial referente à estrutura de tamanhos de espécies classificadas como clímax de dossel, com alta concentração de indivíduos pequenos, estrutura pouco comum para este grupo ecológico, também foi observada para a espécie Alseis backiana, espécie típica de dossel na ilha de Barro Colorado, em Dalling et al. (2001), em que observaram para a espécie atributos de espécies pioneiras e de espécies não pioneiras, entre elas, respectivamente: taxas de germinação maior em áreas de clareiras do que no subosque e baixas taxas de mortalidade no subosque, entre outros fatores. Esses resultados indicam que há lacunas de conhecimento, principalmente no tange à história de vida das espécies, tornando frágeis as classificações das espécies em grupos funcionais.

O grupo das espécies clímax de subosque foi o único que apresentou o padrão esperado, com a distribuição de tamanhos dos indivíduos do tipo "J-invertido". Essa estrutura populacional está comumente associada a espécies com alta capacidade regenerativa, tolerantes à sombra e com baixas taxas de mortalidade devido à alta taxa de recrutamento para as classes de tamanho seguintes (Knight, 1975; Whitmore, 1984; Sato et al., 1994; Tanouchi e Yamamoto, 1995; Meira-Neto e Martins, 2003; Wright et al., 2003; Souza et al., 2012).

O padrão de distribuição diamétrica em forma de "J-invertido" sugere para todas as populações analisadas a presença de representantes em todas as classes de tamanho, porém, com maior densidade nas menores classes. Segundo Alves Júnior et al. (2009), o padrão "J-invertido" indica um balanço positivo entre recrutamento e mortalidade, sendo característico de populações autorregenerativas, uma vez que tal padrão só ocorre quando os indivíduos menores substituem, sucessivamente, os indivíduos adultos na população. Por outro lado, esse padrão também pode indicar ciclo de vida curto com tamanho limitado por características genéticas ou tempo curto de regeneração na mata, ou seja, a floresta está no início do processo de regeneração (Pinto et al., 2007) ou, ainda, limitado potencial de crescimento na área, não alcançando classes diamétricas superiores (Cunha e Silva Junior, 2012), esta última hipótese pode ser aplicada, tanto para as espécies secundárias iniciais e clímax de dossel, que estão aguardando uma situação propícia para se desenvolver. Já as espécies clímax de subosque podem apresentar, além do banco de plântulas/jovens, uma limitação genética de crescimento em diâmetro, decorrente de populações de indivíduos de pequeno porte, mesmo quando adultos (Bernasol e Lima-Ribeiro, 2010).

Os modelos de distribuição testados neste estudo forneceram descrições plausíveis para todas as espécies. A distribuição Log-normal foi a que apresentou o melhor ajuste, este modelo se deve à transformação da variável diâmetro para logaritmo, o que de certa maneira padroniza os valores de diâmetro dentro de uma menor amplitude, diminuindo os valores dos desvios em relação à média (Lima, 2014). O modelo Log-normal, já há algum tempo, tem sido selecionado como o melhor ajuste na descrição da estrutura de florestas temperadas ou manejadas (Bliss e Reinker, 1964; Nanang, 1998; Podlaski, 2008). Estudos recentes na região tropical, em áreas naturais, também têm selecionado esse modelo para a descrição das suas estruturas de diâmetros. Jurinitz (2010), em um estudo no Planalto Atlântico Paulista, selecionou o modelo Log-normal com o melhor ajuste para a população da espécie Guapira opposita; Lima (2014) também selecionou o modelo Log-normal como o melhor ajuste para as populações com maior valor de importância na Caatinga. A seleção deste modelo como o melhor ajuste das distribuições diamétricas indica um contínuo de tamanhos e que as populações se apresentam estáveis, ou seja, o recrutamento compensa a mortalidade ao longo do tempo (Alves Júnior et al., 2009; Lopes et al., 2011; Cunha e Silva Júnior, 2012). 
Apesar de amplamente utilizado, e ter ficado entre o segundo melhor ajuste para a maioria das espécies deste estudo, a limitação do uso do modelo Weibull reside no pressuposto da existência de uma relação linear entre idade e tamanho, sendo a utilização do Weibull meramente fenomenológica quando não há estudos ontogenéticos para que tal relação seja comprovada. A estrutura de tamanhos das espécies estudadas indicou a existência de um banco de plântulas e/ou jovens, consequentemente, não podemos afirmar que exista uma relação linear entre idade e tamanhos (Souza, 2008). Nesse contexto, Lima et al. (2015) alertam que a escolha dos modelos a serem testados deve considerar os pressupostos assumidos na formulação de cada modelo, para se minimizar o risco de um modelo descrever bem um padrão que foi gerado por processos diferentes daqueles assumidos na formulação de tal modelo.

O modelo Exponencial Negativo é tradicionalmente utilizado para descrever as estruturas de tamanho com o formato de "J-invertido", no entanto, foi o terceiro melhor ajuste para quase todas as espécies, apenas a espécie Eugenia expansa apresentou esse modelo como o segundo melhor ajuste. A diferença entre os outros dois modelos testados aqui está no fato de que o modelo Exponencial Negativo apresenta apenas um parâmetro, $\lambda$, que controla a inclinação da distribuição decrescente, este parâmetro é a taxa de ocorrência; a grande vantagem e ampla utilização deste modelo decorrem desta característica, pois quanto mais parâmetros tem um modelo, mais complexa é a sua representação gráfica e interpretação biológica (Lima et al., 2015).

A caracterização da hierarquia de tamanhos de uma determinada população a partir do coeficiente de Gini é uma maneira de se descrever a distribuição de tamanhos sem a interferência dos intervalos de classe (Weiner e Solbrig, 1984). Os valores do coeficiente de Gini foram altos para todas as espécies, indicando baixa equabilidade de tamanhos (alta hierarquia). Tanouchi e Yamamoto (1995), em estudo realizado em uma floresta no sudoeste do Japão, também obtiveram altos valores para o coeficiente de Gini para a maior parte das espécies estudadas, independentemente do seu grupo funcional.
A interpretação biológica do coeficiente de Gini tradicionalmente correlaciona maiores valores de Gini à estabilidade. Entre as populações deste estudo, os maiores coeficientes foram os obtidos para as populações de espécies secundárias iniciais e clímax de dossel, já as espécies de subosque apresentaram os menores valores, indicando que estas espécies estão adaptadas à baixa luminosidade do sobosque e dentre as espécies estudadas são as que apresentam melhor equabilidade de tamanhos, com maior número de indivíduos com tamanhos intermediários.

Ao considerar os grupos ecológicos, a condição discutida acima é reforçada, pois embora o grupo das espécies clímax de subosque apresentem valores altos (acima de 0,5) ainda assim, os valores obtidos são significativamente diferentes dos demais grupos, indicando que as espécies que compõem esse grupo apresentam características comuns de adaptação às condições do subosque. No nosso estudo, a maior desigualdade de tamanhos não foi proporcional à tolerância à sombra, rejeitando a hipótese inicial, a alta hierarquia encontrada pode estar associada à ausência ou à baixa proporção de indivíduos em classes intermediárias e maiores (como observado para as espécies clímax de dossel e secundárias iniciais). Estudos recentes têm destacado que valores muito altos de coeficiente de Gini, nem sempre são indicativos de sustentabilidade (Virillo et al., 2011).

Evidências experimentais destacam que espécies intolerantes e tolerantes à sombra podem germinar sob uma larga extensão de condições ambientais (Pearson et al., 2002), contradizendo as ideias clássicas de Swaine e Whitmore (1988). A seleção ocorreria principalmente nos estádios mais avançados do ciclo de vida, quando as espécies estão crescendo rumo ao dossel (Poorter et al., 2005). Em ambientes nos quais não existem restrições hídricas e o tipo de solo não difere entre as unidades amostrais, como é o caso da área estudada, fatores como a disponibilidade de energia fotossinteticamente ativa passam a ser determinantes nas diferenças de crescimento, na permanência em uma dada classe de tamanho, na probabilidade de recrutamento para classes de maior tamanho e na distribuição espacial das espécies arbustivas e arbóreas dentro das florestas tropicais úmidas (Chazdon, 1988; Poorter et al., 2005). 
As espécies que dependem de luz em algum estádio de seu desenvolvimento ficariam estacionadas na primeira classe de tamanho, ora no banco de plântulas, ora no banco de jovens, à espera de condições de luminosidade apropriadas ao seu desenvolvimento (Augspurger, 1984; Brokaw, 1986; Swaine e Whitmore, 1988; Lima, 2005). A alta concentração de indivíduos no banco de jovens e/ou plântulas sugere a existência de restrições nas taxas de recrutamento entre estas classes devido à baixa disponibilidade de luz (Lima, 2005). Outros fatores bióticos também podem estar associados; como a herbivoria (Schupp, 1988) e competição (Dirzo et al., 1992), no entanto, estudos de dinâmica são necessários para confirmar essas hipóteses.

A partir dos resultados obtidos para as populações estudadas foi possível identificar que as respostas demográficas em relação à luz tendem a ser contínuas com diferenças sutis, ao invés de discretas, no entanto as subdivisões dos grupos ecológicos, embora de grande importância e utilidade, são arbitrárias dentro de um contínuo de caracteres, formando grupos sem limites bem definidos (Denslow, 1980; Swaine e Whitmore, 1988). Portanto, não há uma dicotomia entre espécies tolerantes e intolerantes à sombra, e sim um contínuo de respostas ecofisiológicas e os agrupamentos de espécies ocultam inevitavelmente diferenças inter e intraespecíficas (Alvarez-Buylla e Martínez-Ramos, 1992; Clark e Clark, 1992; Osunkoya et al., 1994; Condit et al., 1996; Dalling et al., 2001; Hubbell, 2001, 2005; Poorter et al., 2006).

\section{CONCLUSÕES}

As espécies agrupadas na categoria de subosque foram as únicas que apresentaram concordância com o modelo de estrutura esperado, por outro lado, as espécies secundárias iniciais e as clímax de dossel apresentaram discordância entre o esperado e o observado, com relação ao tipo de distribuição e à hierarquia de tamanhos. O melhor ajuste selecionado, modelo Log-normal, destaca a alta assimetria positiva e indica a existência de um contínuo de tamanhos. Esses resultados enfatizam a limitação da categorização das espécies em classes discretas de grupos ecológicos e reforça a existência de um contínuo ecológico.
A relação entre a desigualdade de tamanhos e aumento de estabilidade populacional indicou limitações interpretativas, pois não foi obtida uma relação diretamente proporcional entre a tolerância à sombra e o coeficiente de Gini.

As espécies com atributos de tolerância e intolerância à sombra, a depender da fase de vida em que se encontram, como as clímax de dossel e as secundárias iniciais, devem ser prioritárias em estudos de autoecologia.

\section{AGRADECIMENTOS}

Ao CNPq, pela bolsa de estudos concedida nos primeiros dois anos deste estudo. À equipe de apoio do Projeto Temático 40 ha de Parcelas Permanentes. Ao Dr. Bruno Almozara Aranha pelo auxílio nas análises estatísticas. Aos revisores anônimos que colaboraram para o aprimoramento do texto.

\section{REFERÊNCIAS BIBLIOGRÁFICAS}

AUGSPURGER, C.K. Light requirements of neotropical tree seedlings: a comparative study of growth and survival. Journal of Ecology, v. 72, p. 777-795, 1984.

ALVAREZ-BUYLLA, E.R:; MARTINEZ-RAMOS, M. Demography and allometry of Cecropia obtusifolia, a neotropical pioneer tree - an evaluation of the climax pioneer paradigm for tropical rain forests. Journal of Ecology, v. 80, p. 275-290, 1992.

ALVES JUNIOR, F.T. et al. Estrutura diamétrica de um fragmento de Floresta Atlântica em matriz de cana-de-açúcar, Catende, Pernambuco. Revista Brasileira de Engenharia Agrícola e Ambiental, v. 13, n. 4, p. 328-333, 2009.

BAILEY, R.L.; DELL, T.R. Quantifying diameter distributions with the Weibull function. Forest Science, v. 19, p. 97-104, 1973.

BARBOUR, M.G.; BURK, J.H.; PITTS, W.D. Terrestrial Plant Ecology. $2^{\text {nd }}$ ed. Menlo-Park: Benjamim/Cummings, 1987. $634 \mathrm{p}$.

BASKIN, C.C.; BASKIN, J.M. Seeds: ecology, biogeography, and evolution of dormancy and germination. London: Academic Press, 1998. $666 \mathrm{p}$. 
BERNACCI, L.C. et al. O efeito da fragmentação florestal na composição e riqueza de árvores na região da reserva do Morro Grande (Planalto de Ibiúna, SP). Revista do Instituto Florestal, v. 18, p. 123-168, 2006.

BERNASOL, W.P.; LIMA-RIBEIRO, M.S. Estrutura espacial e diamétrica de espécies arbóreas e seus condicionantes em um fragmento de cerrado sentido restrito no sudoeste goiano. Hoehnea, v. 37, n. 2, p. 181-198, 2010.

BLISS, C.I.; REINKER K.A. A lognormal approach to diameter distributions in even-aged stands. Forest Science, v. 10, p. 350-360, 1964.

BOLKER, B.M. Ecological models and data in $\mathbf{R}$. Princeton: Princeton University Press, 2008. 396 p.

Bbmle: tools for general maximum likelihood estimation. R package version 1.0.18. 2016. Disponível em: <https://cran.r-project.org/web/ packages/bbml/index.html>. Acesso em:7 out. 2016.

BONGERS, F. et al. Structure and floristic composition of the lowland rain forest of Los Tuxtlas, Mexico. Vegetatio, v. 74, p. 55-80, 1988.

BROKAW, N.V.L. Seed dispersal, gap colonization, and the case of Cecropia insignis. In: ESTRADA, A.; FLEMING, T.H. (Ed.). Frugivores and seed dispersal. Dordrecht: Dr W. Junk Publishers, 1986. p. 323-331.

CATHARINO, E.L.M. et al. Aspectos da composição e diversidade do componente arbóreo das florestas da Reserva Florestal do Morro Grande, Cotia, SP. Biota Neotropica, v. 6, n. 2, p. 1-28, 2006.

CHAZDON, R.L. Sunflecks and their importance to forest understorey plants. Advances in Ecological Research, v. 18, p. 1-63, 1988.

CHEN, W.J. Tree size distribution functions of four boreal forest types for biomass mapping. Forest Science, v. 50, p. 436-449, 2004.

CLARK, D.A.; CLARK, D.B. Life history diversity of canopy and emergent trees in a neotropical rain forest. Ecological Monographs, v. 62, p. 315-344, 1992.

CLARK, D.B.; CLARK, D.A. Population ecology and microhabitat distribution of Dipteryx panamensis, a neotropical rain forest emergent tree. Biotropica, v. 19, p. 236-244, 1987.
COX, D.R.; OAKES, D. Analysis of survival data monographs on statistics and applied probability. London: Chapman and Hall, 1984. 201 p.

CONDIT, R.; HUBBELL, S.P.; FOSTER, R.B. Assessing the response of plant functional types to climatic change in tropical forests. Journal of Vegetation Science, v. 7, p. 405-416, 1996.

CRAWLEY, M.J. The R book. Chichester: John Wiley \& Sons, 2007. 942 p.

CUNHA, M.C.L.; SILVA JÚNIOR, M.C. Estrutura diamétrica e hipsométrica na Floresta Estacional Semidecidual Montana do Pico do Jabre - PB. Revista Brasileira de Ciências Agrárias, v. 7, n. 2, p. 292-300, 2012.

DALLING, J.W. et al. The unusual life history of Alseis blackiana: a shade-persistent pioneer tree? Ecology, v. 82, p. 933-945, 2001.

DENSLOW, J.S. Gap partitioning among tropical rainforest trees. Biotropica, v. 12, p. 47-51, 1980.

Disturbance and diversity in tropical rain forests: the density effect. Ecological Application, v. 5, p. 962-968, 1995.

DEPARTAMENTO DE ÁGUAS E ENERGIA ELÉTRICA - DAEE. Banco de Dados Hidrológicos. Disponível em: <http://www.hidrologia.daee.sp.gov.br/>. Acesso em: 12 fev. 2016.

DIRZO, R. et al. The effects of gap size and age on the understorey herb community of a tropical Mexican rainforest. Journal of Ecology, v. 80, n. 4, p. 809-822, 1992.

FEELEY, K.J. et al. Do current stem size distributions predict future population changes? An empirical test of intraspecific patterns in tropical trees at two spatial scales. Journal of Tropical Ecology, v. 23, p. 191-198, 2007.

GANDOLFI, S. Estudo florístico e fitossociológico de uma floresta residual na área do Aeroporto Internacional de São Paulo, município de Guarulhos, SP. 1991. 232 f. Dissertação (Mestrado em Biologia Vegetal) - Universidade Estadual de Campinas, Instituto de Biologia, Campinas.

GENTRY, A.H.; TERBORGH, J. Composition and dynamics of the Cocha Cashu "mature" floodplain forest. In: GENTRY, A.H. (Ed.). Four neotropical rainforests. New York: Yale University Press, 1990. p. 542-564. 
HAFLEY, W.L.; SCHREUDER, H.T. Statistical distributions for fitting diameter and height data in even-aged stands. Canadian Journal of Forest Research, v. 7, p. 481-487, 1977.

HARPER, J.L. Population biology of plants. New York: Academic Press, 1977. 892 p.

HARTSHORN, G.S. Neotropical forest dynamics. Biotropica, v. 12, p. 23-30, 1980.

HOBBS, N.T.; HILBORN, R. Alternatives to statistical hypothesis testing in Ecology: a guide to self teaching. Ecological Applications, v. 16, p. 5-19, 2006.

HUBBELL, S.P. Tree dispersion, abundance and diversity in a tropical dry forest. Science, v. 203, p. 1299-1309, 1979.

The unified neutral theory of biodiversity and biogeography. Princeton: Princeton University Press, 2001. 375 p.

Neutral theory in community ecology and the hypothesis of functional equivalence. Functional Ecology, v. 19, p. 166-172, 2005.

; FOSTER, R.B. Canopy gaps and the dynamics of a neotropical forest. In: CRAWLEY, M.J. (Ed.). Plant ecology. Oxford: Blackwell Scientific, 1986. p. 77-96.

. La estructura espacial en gran escala de un bosque neotropical. Revista de Biologia Tropical, v. 35 (supl.), p. 7-22, 1987.

HUTCHINGS, M.J. The structure of plant populations. In: CRAWLEY, M.J. (Ed.). Plant ecology. $2^{\text {nd }}$ ed. London: Blackwell Science, 1997. p. $475-531$.

INSTITUTO BRASILEIRO DE GEOGRAFIA E ESTATÍSTICA - IBGE. Manual técnico da vegetação brasileira. 2. ed. Rio de Janeiro, 2012. 275 p. (Série Manuais Técnicos em Geociências, n. 1).

JURINITZ, C.F. Ecologia das populações de duas espécies arbóreas em fragmentos florestais no Planalto Atlântico Paulista. 2010. 82 f. Tese (Doutorado em Ecologia) - Instituto de Biociências, Universidade de São Paulo, São Paulo.

KNIGHT, D.H. A phytosociological analysis of species-rich tropical forest on Barro Colorado Island, Panama. Ecological Monographs, v. 45, p. 259-284, 1975.
KÖPPEN, W. Climatología: con un estudio de los climas de la tierra. Mexico: Fondo de Cultura Econômica, 1948. 478 p.

LEE, D.W. Irradiance and spectral quality affect Asian tropical rain forest tree seedling development. Ecology, v. 77, p. 568-580, 1996.

LEITE, E.C.; RODRIGUES, R.R. Fitossociologia e caracterização sucessional de um fragmento de Floresta Estacional no Sudeste do Brasil. Revista Árvore, v. 32, n. 3, p. 583-595, 2008.

LIMA, R.A.F. Estrutura e regeneração de clareiras em florestas pluviais tropicais. Revista Brasileira de Botânica, v. 28, n. 4, p. 651-670, 2005.

Ajuste e seleção de modelos na descrição de comunidades arbóreas: estrutura, diversidade e padrões espaciais. 2013. $196 \mathrm{f}$. Tese (Doutorado em Ecologia) - Instituto de Biociências, Universidade de São Paulo, São Paulo.

; BATISTA, J.L.F.; PRADO, P.I. Modeling tree diameter distributions in natural forests: an evaluation of 10 statistical models. Forest Science, v. 61, p. 320-327, 2015.

LIMA, R.B. Distribuição diamétrica e volumetria em vegetação de Caatinga arbustiva-arbórea, Floresta - PE. 2014. 134 f. Dissertação (Mestrado em Ciências Florestais) - Universidade Federal Rural de Pernambuco, Recife.

LOBO-FARIA, P.C. Estratégias adaptativas de espécies arbóreas típicas de ambiente de solo hidricamente saturado: uma abordagem morfológica, bioquímica e ecofisiológica. 1998. 132 f. Tese (Doutorado em Biologia Vegetal) Universidade Estadual de Campinas, Instituto de Biologia, Campinas.

LOPES, S.F. et al. Caracterização ecológica e distribuição diamétrica da vegetação arbórea em um remanescente de Floresta Estacional Semidecidual na fazenda experimental do Glória, Uberlândia, MG. Bioscience Journal, v. 27, n. 2, p. 322-335, 2011.

MEIRA-NETO, J.A.A.; MARTINS, F.R. Estrutura do sub-bosque herbáceo-arbustivo da Mata da Silvicultura, uma floresta Estacional Semidecidual no Município de Viçosa-MG. Árvore, v. 27, n. 4, p. 459-471, 2003. 
MULLER-LANDAU, H.C. et al. Comparing tropical forest tree size distributions with the predictions of metabolic ecology and equilibrium models. Ecology Letters, v. 9, p. 589-602, 2006.

MUNIZ, M.R.A. Estudo do regime de luz nas quatro principais formações fitogeográficas no estado de São Paulo durante o inverno do ano de 2003. 2004. 189 f. Dissertação (Mestrado em Biologia Vegetal) Universidade de Campinas, Campinas.

NANANG, D.M. Suitability of the Normal, Log-normal and Weibull distributions for fitting diameter distributions of Neem plantations in Northerm Ghana. Forest Ecology and Management, v. 103, p. 1-7, 1998.

NUNES, Y.R.F.; PETRERE JÚNIOR, M. Structure and dynamics of a Cariniana estrellensis (Lecythidaceae) population in a fragment of Atlantic Forest in Minas Gerais, Brazil. Rodriguésia, v. 63, n. 2, p. 257-267, 2012.

OLIVEIRA-FILHO, A.T.; CAMISÃO-NETO, A.A.; VOLPATO, M.M.L. Structure and dispersion of four tree population in an area of montane semideciduous forest in southeastern Brazil. Biotropica, v. 28, p. 762-769, 1996.

et al. Diversity and structure of the tree community of a fragment of tropical secondary forest of the Brazilian Atlantic Florest domain 15 and 40 years after logging. Revista Brasileira de Botânica, v. 27, p. 685-701, 2004.

OSUNKOYA, O.O. et al. Influence of seed size and seedling ecological attributes on shade-tolerance ofrain-forest tree species in northern Queensland. Journal of Ecology, v. 82, p. 149-163, 1994.

OTTO, S.P.; DAY, T. A biologist's guide to mathematical modelling in ecology and evolution. Princeton: Princeton University Press, 2007. 732 p.

PANETTA, F.D. Shade tolerance as reflected in population structures of the woody weed, groundsel bush (Baccharis halimifolia L.). Australian Journal of Botany, v. 27, p. 609-615, 1979.

PEARSON, T.R.H. et al. Germination ecology of neotropical pioneers: interacting effects of environmental conditions and seed size. Ecology, v. 83, p. 2798-2807, 2002.
PEARSON, T.R.H. et al. Interactions of gap size and herbivory on establishment, growth and survival of three species of neotropical pioneer trees. Journal of Ecology, v. 91, p. 785-796, 2003.

PINTO, S.I.C. et al. Estrutura do componente arbustivo-arbóreo de dois estádios sucessionais de Floresta Estacional Semidecidual na Reserva Florestal Mata do Paraíso, Viçosa, MG, Brasil. Revista Árvore, v. 31, n. 5, p. 823-833, 2007.

PODLASKI, P. Characterization of diameter distribution data in near-natural forests using the Birnbaum-Saunders distribution. Canadian Journal of Forest Research, v. 38, p. 518-527, 2008.

POORTER, L. et al. Regeneration of canopy tree species at five sites in West African moist forest. Forest Ecology and Management, v. 84, p. 61-69, 1996.

. et al. Beyond the regeneration phase: differentiation of height-light trajectories among tropical tree species. Journal of Ecology, v. 93, p. 256-267, 2005.

.; BONGERS, L.; BONGERS, F. Architecture of 54 moist-forest tree species: traits, trade-offs, and functional groups. Ecology, v. 87, p. 1289-1301, 2006.

R DEVELOPMENT CORE TEAM. R: a language and environment for statistical computing. Vienna: R Foundation for Statistical Computing, 2016. Disponível em: <https://www.r-project.org/>. Acesso em: 4 out. 2016.

RODRIGUES, R.R. 40 ha de Florestas do Estado de São Paulo: uma experiência multidisciplinar. $4^{\circ}$ Relatório Científico. 2006. Disponível em: $<$ http://www.lerf.esalq.usp.br/parrel2005.php $>$. Acesso em: 25 ago. 2015.

SATO, T.; TANOUCHI, H.; TAKESHITA, K. Initial regenerative processes of Distylium racemosum and Persea thunbergii in an evergreen broad-leaved forest. Journal of Plant Research, v. 107, p. 331-337, 1994.

SCHUPP, E.W. Factors affecting post-dispersal seed survival in a tropical forest. Oecologia, v. 76, p. 525-530, 1988.

SILVERTOWN, J.W.; DOUST, J.L. Introduction to plant population biology. Oxford: Blackwell Scientific Pub., 1993. 210 p. 
SOARES JUNIOR, A.A.; VIDAL-TORRADO, P.; SILVA, A.C. Mapeamento ultradetalhado dos solos do Parque Estadual "Carlos Botelho" destinado ao Projeto Diversidade, Dinâmica e Conservação em Florestas do Estado de São Paulo: 40 ha de parcelas permanentes. Piracicaba: Escola Superior de Agricultura "Luiz de Queiroz", 2004. Disponível em: $<$ http://www.lerf.esalq.usp.br/parre12005.php $>$. Acesso em: 25 set. 2015.

SOLBRIG, O.T. Studies on the population biology of the genus Viola. II The effect of plant size on fitness in Viola sororia. Evolution, v. 35, p. 1080-1093, 1981.

SOUZA, F.M. et al. A vegetação da Estação Ecológica de Xitué, Ribeirão Grande-SP: subsídios para o plano de manejo. IF Série Registros, n. 51, p. 5-30, 2014.

SOUZA, P.B.; SOUZA, A.L.; MEIRA NETO, J.A. Estrutura diamétrica dos estratos e grupos ecológicos de uma área de floresta estacional semidecidual, em Dionísio, MG. Revista Árvore, v. 36, n. 1, p. 151-160, 2012.

SOUZA, S.C.P.M. Estratégias de regeneração das espécies arbóreas de um trecho da Floresta Ombrófila Densa Submontana do Parque Estadual de Carlos Botelho, Brasil. 2008. 129 f. Tese (Doutorado em Biologia Vegetal) Universidade Estadual de Campinas, Instituto de Biologia, Campinas.

SPIEGEL, M.R.; STEPHENS, L.J. Estatística. 4. ed. Porto Alegre: Bookman, 2009. 600 p.

SWAINE, M.D.; LIEBERMAN, D.; PUTZ, F.E. The dynamics of tree populations in tropical forest: a review. Journal of Tropical Ecology, v. 3, p. $359-366,1987$.

; WHITMORE, T.C. On the definition of ecological species groups in tropical rain forests. Vegetatio, v. 75, p. 81-86, 1988.

SZTUTMAN, M.; RODRIGUES, R.R. O mosaico vegetacional numa área de floresta contínua da planície litorânea, Parque Estadual da Campina do Encantado, Pariquera Açú, SP. Revista Brasileira de Botânica, v. 25, p. 161-176, 2002.
TABARELLI, M.; MANTOVANI, W. Colonização de clareiras naturais na floresta atlântica do Brasil. Revista Brasileira de Botânica, v. 20, p. 57-66, 1997.

TANOUCHI, H.; YAMAMOTO, S. Structure and regeneration of canopy species in an old-growth evergreen broad-leaved forest in Aya district, southwestern Japan. Vegetatio, v. 117, p. 51-60, 1995.

VIRILLO, C.B. et al. Is size structure a good measure of future trends of plant populations? An empirical approach using five woody species from the Cerrado (Brazilian savanna). Acta Botanica Brasilica, v. 25, n. 3, p. 593-600, 2011.

WEINER, J.; SOLBRIG, O.T. The meaning and measurement of size hierarchies in plant populations. Oecologia, v. 61, p. 334-336, 1984.

WHITMORE, T.C. Tropical rain forest of the Far East. $2^{\text {nd }}$ ed. Oxford: Oxford University Press, 1984. $352 \mathrm{p}$.

. An introduction to Tropical Rain Forests. $\overline{2^{\text {nd }}}$ ed. New York: Oxford University Press, 1990. $296 \mathrm{p}$.

. A review of some aspects of tropical rain forest seedlings ecology with suggestions for further enquiry. In: SWAINE, M.D. (Ed.). The ecology of tropical forest tree seedlings. Paris: Unesco: Parthenon Publishing Group Ltd., 1996. p. 3-39. (Man \& Biosphere Series, v. 18).

WRIGHT, S.J. et al. Gap dependent recruitment, realized vital rates, and size distributions of tropical trees. Ecology, v. 84, n. 12, p. 3174-3185, 2003.

ZAVALA, M.A. et al. An analytical model of stand dynamics as a function of tree growth, mortality and recruitment: the shade tolerance-stand structure hypothesis revisited. Journal of Theoretical Biology, v. 244, p. 440-450, 2007. 